\title{
ESTRATEGIA UNIVERSITARIA BASADA EN HABILIDADES
}

\author{
Rónald Miranda Chavarría* \\ Vanessa Valerio Hernández*
}

La definición de una estrategia global que permita a una universidad alcanzar su misión o su visión de futuro constituye un asunto de importancia trascendental para las autoridades universitarias. En este artículo se propone un modelo denominado estrategia universitaria basada en habilidades como un recurso conceptual y metodológico para el análisis y la definición de estrategias institucionales en universidades. Se plantean los fundamentos, se describen los escenarios estratégicos y se explica el proceso de elaboración de una estrategia, lo cual se ilustra con el caso concreto de la Universidad Nacional, Costa Rica, como parte de un proceso de planificación global.
Defining a global strategy that universities can use in order to fulfill their mission and their vision of the future constitutes a vital task for university authorities. A Skillbased University Strategy model is introduced in this paper as a conceptual and methodological resource to be used in the analysis and definition of institutional strategies within a university. We hereby present the fundamentals of the project, describe its strategic scenarios, and explain the process of strategy creation. This is illustrated through the experience of the global process of planning that the Universidad Nacional, Costa Rica, has gone through.

* Director del Programa Gestión de la Calidad Institucional, Vicerrectoría Académica. Universidad Nacional. Heredia, Costa Rica.

* Licenciada en Planificación. Académica de la Escuela de Ciencias Ambientales y del Programa Gestión de la Calidad Institucional. Universidad Nacional. Heredia, Costa Rica. 
"No hay un sistema perfecto para hacer una estrategia."

H. Mintzberg.

\section{La necesidad de una concepción estratégica en la gestión universitaria}

¿Cómo se garantiza hoy que una universidad cumple con la función social para la cual fue creada? ¿Se están tomando las decisiones adecuadas para que las universidades cumplan su misión de acuerdo con los tiempos actuales y venideros? El actual escenario en el que se da la educación superior es altamente dinámico y competitivo. Las trasformaciones que se han venido dando desde la última década del siglo anterior en el mundo universitario, tales como la masificación de la educación superior (Burdano, 1999; Rama, 2002; UNESCO, 1998a), la internacionalización, la transdisciplinariedad (Burdano, 1999; Ruiz, 2001) o la competencia por las fuentes de financiamiento (Massy, 1996), están llevando a las universidades a definir una clara diferenciación en la oferta académica y a elevar los niveles de calidad en la producción académica y en la gestión universitaria (Bricall, 2000; Guy,2001; UNESCO 1998b). Esta dinámica lleva a las universidades a buscarnuevas estrategias de desarrollo institucional. Consecuentemente, las autoridades universitarias enfrentan un reto importante: la formulación de estrategias institucionales renovadoras.

El proceso de formación de estrategias es un campo de estudio cuyos conceptos y herramientas surgen para ser aplicadas en el contex to empresarial, bajo là premisa de que las organizaciones compiten para obtener cuotas de mercado y para asegurar el triunfo o al menos la supervivencia de la organización en un entorno hostil y globalizado (Hammel y Parlad, 1990; Boyett y Boyett, 1999; Porter, 1980). Aunque las universidades están cada vez más afectadas por un mundo competitivo, no en todos los casos se aplica el supuesto indicado, ya que muchas universidades públicas cuentan con un respaldo jurídico y económico suficiente que asegura la permanencia de la institución. Lo ineludible es que son instituciones llamadas a cumplir de la mejor manera la función que la sociedad les encomendó (Richter, 2002). En tales casos, la estrategia institucional responde a la manera en que una universidad esclarece su vocación y cumple con su misión de manera efectiva y socialmente responsable, la acerca a su visión de futuro y permite diferenciarse de otras instituciones.

La formulación de una estrategia institucional constituye una de las funciones y responsabilidades de las autoridades superiores universitarias, es una tarea compleja, sistemática y rigurosa que requiere de herramientas apropiadas 
para evitar usuales ejercicios superficiales que conllevan a evitar decisiones difíciles para las universidades (Dill, 1994). En eso consiste el aporte de este artículo, presenta un marco conceptual e instrumental para la formulación de estrategias adaptado a la naturaleza de las instituciones universitarias. Se trata de un modelo de estrategia basado en habilidades, en donde este considera que la excelencia en la labor académica y en la gestión de una universidad depende fundamentalmente de habilidades que, mediante sistemas, políticas o estructuras, se consolidan en los funcionarios. La identificación de un sistema de habilidades distintivas en una universidad particular constituye un ejercicio revelador y orientador de la gestión universitaria a largo plazo; por tanto, se sugiere que las autoridades universitarias conozcan y utilicen esta propuesta.

\section{Fundamentos de la estrategia universitaria basada en habilidades}

El concepto de habilidades en el contexto de la definición de estrategias se reporta desde 1980, cuando Porter, el principal influyente en el pensamiento contemporáneo sobre estrategia y competitividad, reconocía que lo significativo del "esfuerzo" estratégico está en las habilidades con las que una empresa se enfrente a las fuerzas externas que determinan la estructura en un sector industrial (Porter, 1980: 23). Sin embargo, el énfasis del concepto de estrategia fue puesto en la búsqueda de posiciones ventajosas con respecto a otros competidores (Boyett, y Boyett, 1999). Posteriormente Stalk, Evans y Shulman (1992) plantean que la estrategia no es solo cuestión de la estructura de los productos y los mercados sino también de la dinámica de los comportamientos de los cuadros dirigentes y operativos ${ }^{1}$; de ahí se plantea que una estrategia sirve para crear un conjunto de habilidades organizativas difíciles de imitar que distinga a una empresa de sus similares. Aunque el concepto constituye un aporte importante al estado de conocimientos acerca de la cuestión estratégica, el desarrollo metodológico e instrumental para diseñar estrategias en la práctica, ha sido limitado.

Mintzber (1988b), al analizar con mayor profundidad el contex to de las organizaciones de profesionales entre las que contempla a las universidades, reconoce que en ellas muchas veces no se establecen estrategias de la manera en que lo recomiendan los expertos como los anteriormente citados. En la práctica, a lo largo del

1 Se entiende que los cuadros dirigentes, es decir, los responsables de las funciones directivas de la organización, deben proyectar un comportamiento coherente en el momento de tomar decisiones. Esas decisiones deben inducir la acción de los funcionarios operativos; es decir, aquellos que en forma directa realizan las tareas formadoras de los productos o servicios principales (en la organización académica son los académicos y los funcionarios de apoyo). En la trama de decisiones y acciones operativas, lo que está a prueba son las habilidades de todos los miembros de la organización. 
tiempo se establecen patrones en la forma de tomar decisiones que finalmente constituyen las estrategias. Adicionalmente, Mintzberg advierte que en las organizaciones de profesionales, la calidad del trabajo operativo depende de las habilidades con las que cuentan los profesionales que conforman la organización.

A partir de esos conceptos, resulta conveniente que en el contexto universitario la formulación de estrategias se haga de manera explícita y metódica de forma tal que consolide las habilidades que hacen a una universidad distinguirse de otras. Lamentablemente, bajo esta concepción los recursos metodológicos e instrumentales son limitados. Por eso se propone asumir la noción de estrategia universitaria basada en habilidades para orientar la gestión universitaria hacia el desarrollo de las habilidades que converjan en el cumplimiento de la misión institucional y en el logro de la visión de futuro. La estrategia así definida se construye mediante un proceso sencillo, aunque laborioso. Se trata de un recurso analítico - sintético que permite establecer una estrategia que conjuga dos elementos: una posición genérica, entendida como la ubicación dentro de uno de los escenarios propuestos en la Figura 1 y un sistema de habilidades distintivas.

\section{Supuestos}

En la construcción de una estrategia universitaria basada en habilidades se asumen varios supuestos. El primero de ellos es concebir a la universidad como una organización de profesionales académicos que cumple su misión a partir de los talentos de estos, lo cual se manifiesto en las habilidades para construir y socializar el conocimiento. Lo distintivo y la competitividad de una universidad no reside tanto en la suma de los conocimientos de sus académicos, como en las habilidades ampliamente utilizadas por estos en los procesos pedagógicos, investigativos y de extensión, así como en los procesos de gestión universitaria.

El segundo supuesto es el reconocimiento de que la puesta en práctica de toda estrategia enfrenta adversidades o fuerzas contrarias al propósito estratégico. Las adversidades pueden ser endógenas (debilidades) o exógenas (amenazas), pero ambas actúan en dirección contraria al logro de la visión o de algún objetivo estratégico. Consecuentemente, una estrategia debe construirse a partir de la identificación de las adversidades críticas y de las habilidades que hace posible superar esas adversidades. El efecto lógico de este proceso es que una estrategia llega a tener una importante inmunidad en su "código genético". En palabras directas, desde la concepción de una estrategia, esta debe llevar una importante carga de información acerca de las posibles adversidades que podrá encontrar, y debe prever las posibles respuestas a esas adversidades. 
El tercer supuesto es la aceptación del concepto porteriano de "estrategia genérica", pero no como categoría absoluta sino que adaptado como síntesis facilitadora y orientadora ante grandes escenarios estratégicos. La estrategia basada en habilidades aporta también una definición sintética de las estrategias básicas o genéricas que puede seguir una institución universitaria para alcanzar la visión. Particularmente se trata de cuatro escenarios estratégicos o tipos de estratégias que los denominamos estrategia de consolidación, de potenciación del talento humano, de gestión y política y, de cambio hábil.

El cuarto supuesto establece que la materialización de la estrategia se logra a partir del conjunto de las habilidades que hacen a una universidad sobresalir respecto a otras. Los profesionales, tanto académicos como administrativos, poseen múltiples habilidades, pero solo una cuantas son decisivas para la estrategia, esas deben desarrollarse hasta que se instituyan y sean del dominio de una proporción importante de los funcionarios universitarios. Es por eso que la estrategia basada en habilidades llega a precisar un sistema de habilidades distintivas que son las llamadas a dar sostenibilidad a las estrategias.

\section{Los escenarios estratégicos}

En la puesta en ejecución de cualquier estrategia, se ponen en juego las dos fuerzas ya mencionadas: las adversidades y las habilidades con las que se superan las adversidades y se alcanza la visión. La combinación analítica del grado de complejidad de las adversidades y de las habilidades permite identificar los cuatro escenarios estratégicos básicos (Figura 1).

En los casos en que alcanzar una visión no demanda grandes adversidades ni tampoco demanda complejas habilidades, entonces se puede seguir una estrategia de consolidación institucional; pero, si se hace necesario desarrollar e instituir nuevas o más complejas habilidades, se puede seguir una estrategia de potenciación del talento humano. Si la visión de futuro se podría ver comprometida por altas adversidades, pero no demanda nuevas ni complejas habilidades, se puede seguir una estrategia de mejora de la gestión conjugada con la conciliación de la voluntad política; por último, si se requiere desarrollar nuevas y más sofisticadas habilidades, entonces la estrategia conveniente consiste en manejar el proceso de cambio institucional inteligentemente y de manera hábil.

2 Porter (1980 y 1986: pp. 55 -67) establece tres estrategias genéricas: liderazgo en costos, diferenciación y enfoque. En su concepto resultan intemamente consistentes para crear una posición defendible a largo plazo y sobresalir frente a otros competidores. La diferencia de fondo que establecemos con Michael Porter consiste en que ese autor concibe sus estrategias para ganar posición en un mercado en que varias empresas son rivales dentro de una misma industria, en tanto que nuestra propuesta de estrategia en habilidades está concebida en función del logro de la vocación institucional, entendida comola misión social adoptada, lo cual es útil en un contexto institucional dondela búsqueda del sentido de identidad y de realización son importantes factores movilizadores en los miembros de la organización. 


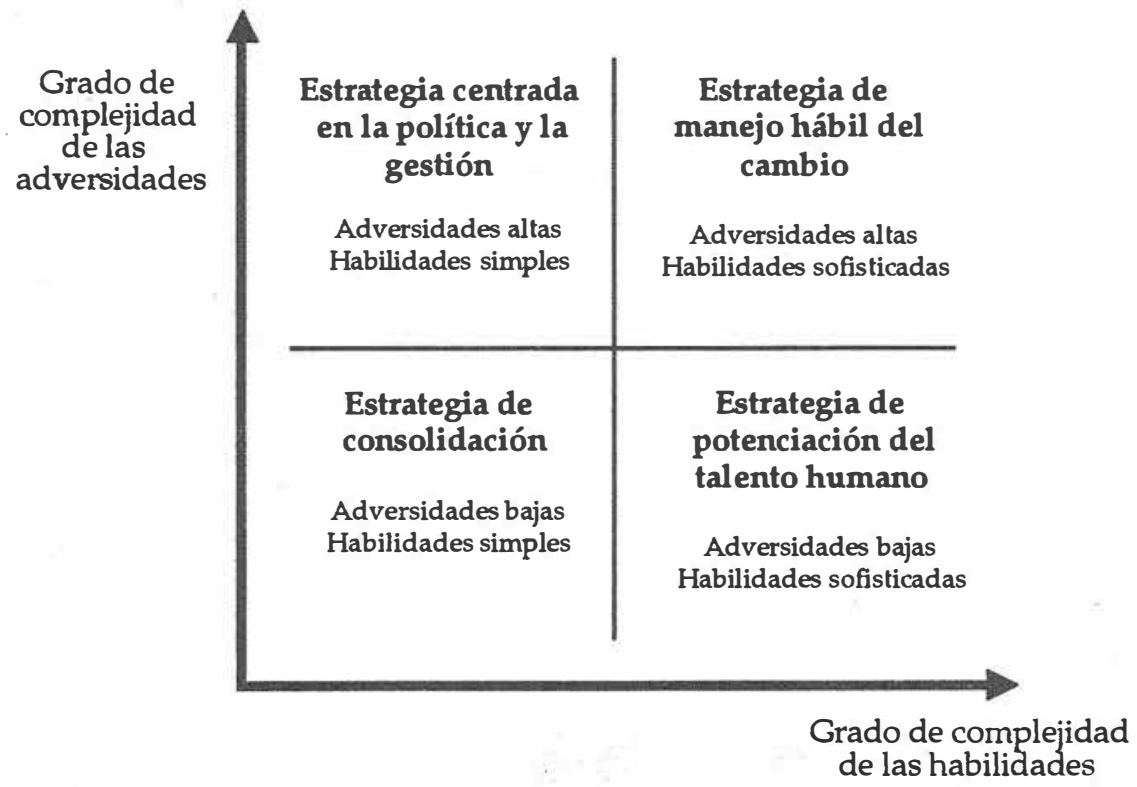

Figura 1. Escenarios estratégicos genéricos basados en habilidades y adversidades.

A continuación se explican las cuatro estrategias citadas.

\section{Eestrategia de consolidación}

Es el camino que conviene seguir si la visión de futuro está conformada por elementos que se pueden alcanzar con las estructuras, las políticas y los procesos establecidos y sin más habilidades fundamentales que son del dominio de la mayoría de los profesionales de la universidad. Es una estrategia conveniente cuando la visión se logra, manteniendo las fortalezas alcanzadas y defendiendo las conquistas adquiridas. Es adecuada si la institución goza de estabilidad en cuanto a las demandas externas, en las condiciones presupuestarias y en materia de recursos humanos. El riesgo de esta estrategia es que puede causar estancamiento y que la institución sea percibida como conservadora.

\section{Estrategia de potenciación del talento humano}

Es una estrategia indicada en los casos en que la visión es alcanzable con las actuales estructuras, procesos y políticas, pero requiere del desarrollo de habilidades especializadas que no están al alcance de la mayor parte de los profesionales con que cuenta la institución. Bajo tales condiciones se requiere invertir en un esfuerzo de capacitación orientada a desarrollar las habilidades que posibiliten el logro de la 
visión, es una estrategia de bajo riesgo. Una institución que opte su desarrollo a partir del desarrollo del talento, puede ser percibida como una entidad de alta experticia.

\section{Estrategia de gestión con voluntad política}

Es una estrategia adecuada si la visión contiene desafíos que no se pueden alcanzar con las actuales políticas, estructuras o sistemas, pero la institución cuenta con las habilidades requeridas. En un escenario así es fundamental alcanzar la voluntad política para adoptar las decisiones necesarias para eliminar las barreras internas y entablar las negociaciones externas que permitan, al menos, contener las amenazas. Paralelamente, son decisivas las mejoras en la eficacia y la eficiencia en la gestión superior y en los servicios de apoyo. La vulnerabilidad de esta estrategia reside en que los intereses políticos divergentes pueden permanecer latentes y surgir con algún cambio coyuntural. Bien llevada, esta estrategia permite que la institución se perciba como una entidad eficiente y con una buena calidad en el servicio; de lo contrario, puede degenerar en proyecciones y percepciones de alto burocratismo.

\section{Estrategia del manejo del cambio con habilidad}

Es una estrategia apta si la visión compromete la generación de nuevas estructuras, procesos, políticas y normas; además, requiere de habilidades aún no adquiridas por la mayoría de los profesionales responsables de la gestión y de los procesos fundamentales. Las decisiones son complejas, pues requieren de una comprensión muy adecuada de los procesos de cambio organizativo, tanto en los aspectos estructurales como en los culturales. Lo esencial de esta estrategia radica en la habilidad para gestionar acertada e inteligentemente los cambios, lo cual requiere desarrollar nuevos paradigmas mentales, nuevas actitudes y hábitos en las personas. El riesgo reside en desestimar los conocimientos requeridos en la conducción de cambios estructurales y culturales combinadamente. Esta estrategia puede generar la percepción de la institución como una entidad líder por su capacidad de actualización.

\section{Indicadores de ejecución}

La puesta en ejecución de cualquier estrategia basada en habilidades requiere de la vigilancia cuidadosa de dos grupos de indicadores de cumplimiento. El primero viene dado por indicadores que corresponden al tipo de estratégia general que se acaban de describir, estos se describen en el Cuadro 1 . El segundo grupo de indicadores es más específico y deben elaborarse en función del conjunto de habilidades distintivas identificadas. Por ejemplo, si una habilidad 
Cuadro 1. Caracterización de las estrategias genéricas incluyendo los indicadores claves de la puesta en ejecución.

\begin{tabular}{|c|c|c|c|c|}
\hline 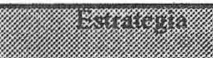 & 1) & (6) & 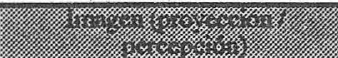 & mos \\
\hline Consolidación & $\begin{array}{l}\text { Consolidar y mantener el } \\
\text { nivel de logros, } \\
\text { previniendo el riesgo de } \\
\text { estancamiento. }\end{array}$ & $\begin{array}{l}\text { La visión de la institución se } \\
\text { puede alcanzar con las } \\
\text { estructuras y políticas } \\
\text { actuales y mediante el uso de } \\
\text { las habilidades disponibles } \\
\text { (adversidades bajas y } \\
\text { habilidades simples). }\end{array}$ & $\begin{array}{l}\text { Institución conservadora o de } \\
\text { mucha tradición. }\end{array}$ & $\begin{array}{l}\text { - } \mathrm{N}^{\circ} \text { de servicios calificados satisfactoriamente } \\
\text { por los usuarios. } \\
\text { - } \mathrm{N}^{\circ} \text { de estudiantes de primer ingreso / } \mathrm{N}^{\circ} \text { de } \\
\text { graduados. } \\
-\mathrm{N}^{\circ} \text { líderes de proyectos que asisten a eventos } \\
\text { de actualización / total de lideres de proyectos. } \\
\text { - } \mathrm{N}^{\circ} \text { anual de productos académicos. }\end{array}$ \\
\hline $\begin{array}{l}\text { Potenciación del } \\
\text { talento humano }\end{array}$ & $\begin{array}{l}\text { Desarrollar capacidades } \\
\text { requeridas y optimizar la } \\
\text { ubicación del recurso } \\
\text { humano. }\end{array}$ & $\begin{array}{l}\text { La visión se puede alcanzar } \\
\text { manteniendo las estructuras y } \\
\text { sistemas, pero modiante } \\
\text { nuevas habilidades } \\
\text { (adversidades bajas y } \\
\text { habilidades sofisticadas). }\end{array}$ & $\begin{array}{l}\text { Institución con alta } \\
\text { experticia. }\end{array}$ & $\begin{array}{l}\text { - } \mathrm{N}^{\circ} \text { horas funcionario en capacitación en } \\
\text { función de los cargos. } \\
-\mathrm{N}^{\circ} \text { de funcionarios con cormpetencias } \\
\text { evaluadas según sistema de puestos. } \\
\text { - } \mathrm{N}^{\circ} \text { funcionarios reclasificados en puestos / } \\
\text { total de funcionarios. }\end{array}$ \\
\hline Política y gestión & $\begin{array}{l}\text { Mejorar los procesos de } \\
\text { decisión política, asegurar } \\
\text { la voluntad política y } \\
\text { mejorar la efectividad de la } \\
\text { gestión. }\end{array}$ & $\begin{array}{l}\text { El logro de la visión no } \\
\text { requiere de habilidades } \\
\text { adicionales, pero requiere de } \\
\text { nuevas políticas y cambios en } \\
\text { los sistemas institucionales. } \\
\text { (Adversidades altas y } \\
\text { habilidades simples). }\end{array}$ & $\begin{array}{l}\text { Institución con servicios } \\
\text { eficientes. Burocratismo. }\end{array}$ & $\begin{array}{l}\text { - } \mathrm{N}^{\circ} \text { de procesos de decisión descentralizados } \\
\text { mediante política explícita. } \\
\text { - Indicador de transparencia (combina } \mathrm{N}^{\circ} \text { de } \\
\text { autoridades que rinden cuentas / Total de } \\
\text { autoridades + } \mathrm{N}^{\circ} \text { de unidades con criterios de } \\
\text { decisión preestablecidos / Total de unidades + } \\
\mathrm{N}^{\circ} \text { de resoluciones que anexan información } \\
\text { comparativa / Total de resoluciones). } \\
\text { - Horas mensuales dedicadas al análisis de la } \\
\text { estrategia o agenda de largo plazo. } \\
\text { - } \mathrm{N}^{\circ} \text { autoridades involucradas en el análisis } \\
\text { estratégico / Total de autoridades. } \\
\text { - } \mathrm{N}^{\circ} \text { quejas o denuncias mensuales sobre la } \\
\text { gestión (buzones o intranet). }\end{array}$ \\
\hline Cambio hábil & $\begin{array}{l}\text { Adecuar la estructura y } \\
\text { los sistemas, manejar la } \\
\text { cultura institucional y } \\
\text { cultivar nuevos } \\
\text { paradigmas y hábitos. }\end{array}$ & $\begin{array}{l}\text { La visión compromete la } \\
\text { generación de nuevos } \\
\text { sistemas, y políticas y el } \\
\text { desarrollo de nuevas } \\
\text { habilidades. (Adversidades } \\
\text { altas y habilidades } \\
\text { sofisticadas). }\end{array}$ & Institución actualizada. & $\begin{array}{l}\text { - } \mathrm{N}^{\circ} \text { de macro procesos rediseñados en función } \\
\text { del modelo de gestión. } \\
\text { - } \mathrm{N}^{\circ} \text { autoridades formadas para gestionar el } \\
\text { cambio / Total de autoridades. } \\
\text { - } \mathrm{N}^{\circ} \text { participantes en actividades de renovación } \\
\text { / Total de funcionarios (por semestre). }\end{array}$ \\
\hline
\end{tabular}

Fuente: Elaboración propia. 
distintiva es concentrarse en programas estratégicos para el desarrollo, se requiere de indicadores del impacto de los proyectos al desarrollo de la sociedad.

La transformación de la estrategia en indicadores permite pasar del valor inspirador de la visión y de lo orientador de la estrategia a las acciones y los resultados concretos. Los indicadores finalmente se pueden organizar dentro de un sistema hipotético de relaciones causa efecto, clasificándolos en las dimensiones o perspectivas del aprendizaje de los funcionarios, de los avances en procesos internos, del aporte a los estudiantes y beneficiarios externos y de las finanzas institucionales (Kaplan, 2002).

\section{El proceso de formulación de la estrategia Construir Visión}

La visión se refiere a la clarificación mental de la imagen de la universidad que se quiere desarrollar en el futuro es el punto de partida Aunque hay muchas formas de hacerlo, en el contexto universitario, la visión surge de las aspiraciones queenuna comunidad de académicos, se hacen explícitas de manera organizada. En comespondencia con esta premisa, la visión prospectiva de una universidad debe preservar el espíritu de la filosofia básica de la institución, debe estimular el progreso en lo institucional y en lo académico y debe adaptar a la universidad al nuevo escenario histórico.

Construir una visión es básicamente un proceso de síntesis en el cual se llegan a establecer un conjunto de ideas fuerza o elementos de visión que describen la imagen de la institución hacia la cual se orientarán los esfuerzos estratégicos. Entre los elementos de la visión hay varios niveles o categorías de ideas: desde lo inspirador hasta lo que orienta los procesos operativos o académicos centrales, pasando por lós que dan dirección política a la institución y por aquellos que guían la gestión. A partir de esos elementos se realiza la identificación de la estrategia institucional.

\section{El análisis de adversidades y de habilidades}

Conforme se logra un consenso acerca de las principales ideas que deben formar parte de la visión se analiza globalmente sus implicaciones mediante la identificación de riesgos y requerimientos decisivos. Es un análisis importante no solo porque da una idea de la viabilidad de la visión, sino porque refuerza el compromiso para su puesta en ejecución y, además, establece el tipo de estrategia institucional más conveniente de adoptar (Miranda y Carranza, 2002).

El análisis se hace separadamente con cada elemento de la visión. Un primer ejercicio del análisis consiste en pronosticar todas las adversidades, barreras, dificultades o impedimentos críticos posibles. Se trata de inventariar todo aquello que dificulte el logro de una aspiración. Las adversidades pueden ser internas (debilidades) o externas (amenazas), pero conviene considerar las que 
verdaderamente resulten críticas para alcanzar una aspiración. Posteriormente, mediante un proceso de síntesis, se identifica un conjunto de habilidades esenciales, es decir, aquellas cosas que la institución requiere hacer de manera sobresaliente, sistemática y sostenida para lograr el elemento de visión analizado.

Es necesario hacer una clasificación de las adversidades y habilidades, cuando un conjunto de adversidades se mantienen dentro del patrón normal de barreras, problemas o contingencias que se pueden resolver dentro de las actuales estructuras, políticas o sistemas, entonces se consideran como adversidades bajas. Pero si la experiencia demuestra que no pueden ser resueltos con las políticas, estructuras o sistemas vigentes, entonces se catalogan como adversidades altas.

Las habilidades se discriminan con atributos diferentes. Se consideran simples, aquellas habilidades que forman parte de las competencias generales de cualquier categoría de profesionales y que permiten ejecutar las actividades básicas, académicas o de gestión. Las habilidades se consideran sofisticadas cuando se basan en conocimientos o entrenamientos especializados a los cuales no todos los profesionales acceden. Para instituir las habilidades sofisticadas se requiere de una inversión sistemática y sostenida en formas avanzadas de desarrollo profesional que deben ser coherentes con la visión. Por ejemplo, si una universidad tiene todos sus sistemas diseñados para ofrecer carreras presenciales y en el futuro quiere ofrecer una docencia a través de internet, tendrá que desarrollar habilidades sofisticadas. En un caso así, el atributo sofisticado, no está dado por el carácter "avanzado" de tecnología, sino más bien en el hecho de que para que la nueva oferta funcione bien, las nuevas habilidades deben instituirse tanto en los docentes, como en los servicios documentales, los sistemas de registro y todos los procesosconexos con la gestión curricular. Para eso se requiere invertir en el desarrollo o la adquisición de las nuevas habilidades mediante programas de capacitación y por la contratación de personas que posean dichas habilidades.

Una vez clasificadas las adversidades y las habilidades asociadas a cada elemento de la visión, se elabora un mapa perceptual como el que se ilustra en la Figura 2.

El primer aspecto de la estrategia institucional queda establecido por la estrategia genérica que corresponde a la ubicación predominantemente de los elementos de visión en los mapas estratégicos. Ahora es posible comprender mejor el significado de la estrategia en el contexto institucional. Es determinante que en esta fase se logre una convicción respecto de la conveniencia de seguir una estrategia de consolidación, de potenciación humana, de política y gestión o de manejo hábil del cambio. La pregunta que surge es ¿qué se debe hacer para poner en práctica esa estrategia? Surge entonces un segundo aspecto de la estrategia: el sistema de habilidades, que guía las decisiones y las acciones mediante las cuales se pone en ejecución la estrategia institucional. 
Elemento de visión: ... ser una universidad con una administración moderna...

\author{
Dificultades críticas \\ Altas / bajas \\ Habilidades distintivas \\ Simples / sofisticadas
}

(tendremos que enfrentar la)

rigidez de los reglamentos internos.

(necesitamos desarrollar la habilidad de)

Alta

gestionar basados en principios de efectividad, en conocimientos e información.

Sofisticada

Habilidades

\begin{tabular}{|c|c|c|c|}
\hline & & Simples & Sofisticadas \\
\hline \multirow{2}{*}{ Dificultades } & Altas & $\begin{array}{l}0 \\
0 \\
0\end{array}$ & \\
\hline & Bajas & $\stackrel{0}{0}$ & \\
\hline
\end{tabular}

Figura 2. En la parte superior se muestra un ejemplo de una matriz utilizada en la identificación y clasificación de as adversidades y las habilidades. Nótese que se hace para cada elemento dela visión. Abajo se muestra un mapa estratégico perceptual en el que cada punto representa a un par ordenado de una dificultad y su respectiva habiidad asociada. La ilustración corresponde a un ejemplo en el que se identificaron diez dificultades concomitantes: con diez habilidades. Todas las habilidades se consideraron simples; tres adversidades se clasificaron como bajas y siete como altas. Este mapa indica la conveniencia de adoptar una estrategia centrada en la política y la gestión. Cuandose completa el análisis para todos los elementos de visión se tiene un conjunto de mapas que muestran un patrón a partir del cual se puede llegar a concluir el tipo de estrategia más conveniente para alcanzar la visión.

Fuente: Miranda, R; Valerio, V; Carranza, C.F; Gillies, A.L. y Vega, M. (2003). Taller: Estrategia institucional. Guía del participante.

\title{
El sistema de habilidades
}

A partir de las habilidades previamente esclarecidas, se hace un trabajo minucioso para detectar cuáles de ellas resultan determinantes para alcanzar lo esencial de la visión. Dado que la visión reúne diversas ideas, es preciso establecer cuál de ellas expresa el gran desafío que la institución se plantea. Alrededor del elemento inspirador o de ese desafío, se construye un tejido de habilidades, partiendo de las que resulten esenciales para alcanzar el desafío que plantea la 
La visión de futuro se expresa a partir de una idea fundamental e inspiradora, la cual es acompañada de otras ideas que orientan el rumbo político de la institución, luego se identifican otros elementos que orientan lo académico y la gestión.

visión. Las habilidades identificadas se agrupan por afinidad y se trazan relaciones de interdependencia hasta establecer unos cuantos núcleos fundamentales, bien diferenciados entre sí, que reflejen lo que la universidad ha de hacer de manera sobresaliente y sostenida. Se obtiene así lo que denominamos habilidades distintivas porque son las que en definitiva le permiten a una universidad diferenciarse de otras universidades. En síntesis, las habilidades distintivas se caracterizan por tener una relación directa con la visión, son de naturaleza diferente entre sí, pero se refuerzan mutuamente.

En vista de que dicho sistema de habilidades se construye a partir de la visión, la cual es específica para cada universidad, resulta comprensible que hipotéticamente puede suceder que al menos dos instituciones adopten una misma estrategia genérica, pero la manifestación concreta de la estrategia puede resultar completamente diferente, pues las habilidades en juego responden a visiones y desarrollos diferentes. Esto nos lleva a establecer que las estrategias basadas en habilidades consisten en un arreglo único para cada universidad, por tanto como sistema no son replicables; es decir, ninguna otra universidad podrá tener el mismo sistema de habilidades puesto que otras universidades pueden tratar de imitar o replicar una o varias habilidades, pero el conjunto de todas las habilidades distintivas no solo es difícil imitarlo sino que no tiene sentido hacerlo, pues cada universidad tiene una visión diferente y por tanto los sistemas de habilidades requeridos necesariamente son diferentes.

Las habilidades distintivas tienen un poder orientador estratégico, por tanto funcionan como objetivos estratégicos. Es decir, a partir de ellas se pueden desplegar acciones, factores claves de éxito e indicadores de logro, los cuales son componentes claves de la planificación y la administración estratégica. Es por eso que la incorporación del concepto de estrategia universitaria basada en habilidades ofrece un marco conceptual y perceptual que enriquece la gestión universitaria de manera indisoluble con los propósitos académicos. Es decir; contribuye a superar la visión fragmentada y dicotómica de la gestión universitaria tradicional, en la cual hay una fuerte separación entre las funciones académicas y las administrativas. 


\section{La experiencia en la Universidad Nacional}

La Universidad Nacional es una universidad pública financiada principalmente con recursos provenientes del Estado costarricense. Se ubica en la ciudad de Heredia, Costa Rica. Además, cuenta con dos sedes regionales, una en la Región Chorotega, al norte del país, a unos 220 km. de la ciudad de Heredia; la otra en la Región Brunca, al sur del país a unos $140 \mathrm{~km}$. de la sede central. Recientemente como parte de esta, se creó la subsede Coto en Ciudad Neilly en la zona fronteriza con Panamá.

Los datos de diciembre del 2003 dan cuenta de una oferta académica conformada por 209 carreras de grado y pregrado y 40 maestrías, doctorados y especialidades. La población universitaria es aproximadamente de 13.500 estudiantes y 1500 funcionarios académicos y administrativos. Las actividades académicas incluyen la docencia, la investigación, la extensión y la producción que abarca la creación artística y literaria. Hay un amplio espectro de disciplinas, temas, dimensiones y ejes temáticos; sin embargo, las áreas estratégicas de conocimiento principales son la educación, el ambiente y uso del territorio, las ciencias y tecnologías, la sociedad y el desarrollo humano, el arte y el desarrollo de la cultura y la salud y la calidad de vida.

El estatuto orgánico de la institución establece los planes académicos anuales y de mediano plazo (de al menos cinco años). También hace referencia al Plan Global Institucional de mediano plazo cuya aprobación corresponde a la Asamblea de Representantes de la Universidad. Durante los últimos años no se ha contado con planes quinquenales que orienten el desarrollo institucional, únicamente se han venido formulando los planes anuales operativos. Esto ha generado un patrón de desarrollo institucional basado en mejoras y en la resolución casuística de problemas, pero sin un direccionamiento estratégico.

Para el año 2002 el desafío planteado fue cómo elaborar un plan suficientemente consensuado y participativamente construido que comprometa a la institución con una renovación significativa, que eleve los niveles de calidad tanto en lo académico como en la gestión institucional, y que pueda tener una viabilidad política dentro de las condiciones económicas y culturales de la institución.

\section{Construcción de una visión compartida}

La construcción de la visión ha sido un prolongado desarrollo en el que se han visualizado los procesos académicos: la investigación, la docencia y la extensión, además del modelo de gestión institucional. Esto se realizó en espacios de participación en los que se contó con académicos destacados en todos los 
campos del saber, con estudiantes distinguidos y con autoridades universitarias de los diferentes niveles. En esas actividades se conjugaron momentos de prospección y de análisis estratégico en forma interactiva. En todas las actividades se invitó a conferencistas o se organizaron mesas redondas para que los participantes tomen conciencia del cambiante escenario histórico y de los factores del entorno nacional e internacional que influyen en el futuro de las universidades. Una vez alimentado el pensamiento en torno al exterior (dimensión espacial) y al futuro (dimensión temporal) de la Universidad, se identifican los deseos y aspiraciones que los funcionarios universitarios expresan aquí y ahora acerca del futuro de la institución. Las múltiples ideas y propuestas se priorizaron, se sistematizaron y se sintetizaron hasta llegar a un conjunto de ideas que expresan deseos colectivos y desafíos que generan un compromiso compartido.

La visión de futuro se expresa a partir de una idea fundamental e inspiradora, la cual es acompañada de otras ideas que orientan el rumbo político de la universidad, luego se identifican otros elementos que orientan lo académico y la gestión. El enunciado de la visión satisface además otras tres condiciones que se preestablecieron. Primero, se preserva el núcleo filosófico de la Universidad Nacional: ser la universidad necesaria, lo que conjuga la excelencia académica con el compromiso social: "La Universidad Nacional forma profesionales de excelencia con conocimientos holísticos, propositivos e innovadores capaces de contribuir significativamente a un desarrollo humano equitativo y sostenible". La segunda condición establece la adaptación a los nuevos tiempos, en ese sentido expresa, por ejemplo que la docencia "utiliza un modelo pedagógico innovador e incorpora el uso de las nuevas tecnologías para responder a los avances del conocimiento". Finalmente, asegura el progreso institucional al orientar la gestión hacia la desconcentración y el uso de nuevas tecnologías de información (Universidad Nacional, 2003: 30).

\section{El análisis estratégico}

El proceso de análisis estratégico en la práctica se fue introduciendo de manera simultánea con la construcción de la visión. En un primer momento se utilizó una técnica perceptual con la intención de confrontar una realidad deseada con la actual para configurar una visión realista y motivadora a la vez (Van Vucht Tijssen, 2002). Sin embargo, rápidamente se perfeccionó la técnica y se introdujo el análisis de adversidades y habilidades, que permitió reconocer la complejidad y los riesgos asociados a cada elemento de la visión. Como resultado se concluyó que para lograr la visión propuesta conviene seguir una estrategia de gestión de cambio.

El paso siguiente consistió en sistematizar las habilidades necesarias para alcanzar los diferentes elementos de la visión. El resultado es que hay cinco 
habilidades distintivas (Figura 3); cada una de ellas se desagrega en un conjunto adicional de habilidades de segundo orden, que le dan contenido y proveen una especificidad operativa.

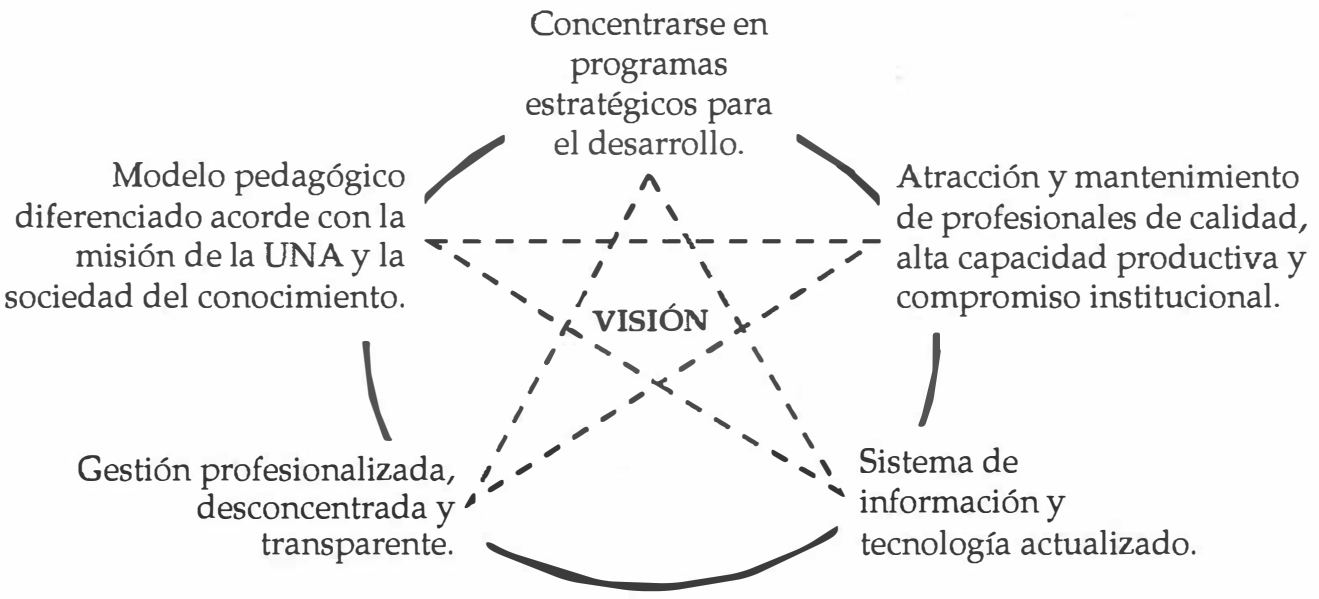

Figura 3: Habilidades distintivas requeridas para el logro de la visión de la Universidad Nacional.

A partir de la primera propuesta de estrategia, que incluía el sistema de habilidades distintivas más otras necesarias para el logro de la misión, se establecieron objetivos, políticas y acciones de carácter estratégico. Esta es una fase que establece la forma de operacionalizar la estrategia institucional. En la práctica se vio realimentada por información proveniente de otros procesos institucionales orientados al cambio, por ejemplo, el Congreso Universitario.

\section{El análisis político}

En esta fase participaron las autoridades que forman parte de los denominados órganos de conducción superior, se trata del Consejo Universitario, la Rectoría y su Gabinete y el Consejo Académico que está constituido principalmente por los decanos; participaron además, los representantes del Gobierno Estudiantil que forman parte de los órganos colegiados.

En el momento en que la visión, la estrategia y su propuesta de operacionalización fue sometida a discusión por parte de las autoridades oficiales, se muestra una fuerte preocupación por la viabilidad política de la estrategia. Una importante consideración es si en la estrategia formulada se ven reflejados los intereses de los distintos sectores universitarios. Así surge una dinámica en la que las estrategias originalmente identificadas se replantean: unas 
se segregan, otras se fusionan y se incorporan otras nuevas con la intención de hacer más visibles los temas de interés para cada sector universitario. A partir de este momento, las estrategias que estaban expresadas en términos de habilidades distintivas, pasan a expresarse más como tópicos que pueden estimular la adhesión de los distintos sectores universitarios a la estrategia institucional.

En esta fase de análisis político, se identifican también los mecanismos para la aprobación formal del Plan Global Institucional que contiene la estrategia. Además, se analizan los requerimientos necesarios para generar compromiso político necesario para la sostenibilidad de la estrategia. La culminación de esta fase se logró cuando el Plan Global fue finalmente aprobado por la Asamblea de Representantes. Por tratarse de una decisión colegiada, siempre se hacen observaciones y enmiendas al Plan, pero lo sustantivo de la estrategia construida por el método aquí descrito, resulta aceptada por quienes toman la decisión final.

\section{Previsión para la ejecución}

Una vez formulada la estrategia se manifiesta la preocupación por el riesgo de fracaso al ponerla en ejecución. Como previsión, en el caso de la Universidad Nacional se incorporaron dos instrumentos de gestión para ser desarrollados. Uno es el plan táctico, definido en este caso como un instrumento de planificación que detalla la forma en que se ejecutarán las acciones estratégicas en lapsos de dos o tres años, incluyendo los responsables y los medios.

El segundo se denominó Sistema de Conducción Integral ${ }^{2}$, el cual facilita el monitoreo y el control estratégico, mediante indicadores clave que señalan el grado de cumplimiento de los objetivos estratégicos ${ }^{3}$. El sistema de conducción integral coadyuva a la toma de decisiones, facilita la rendición de cuentas y contribuye al seguimiento y a la evaluación integral de la estrategia (Kaplan y Norton, 2002b).

Adicionalmente, se identificaron varios temas generadores que impulsan la ejecución de la estrategia en el corto plazo. Algunos de esos temas constituyen experiencias piloto, nuevas políticas o esfuerzos institucionales; por ejemplo, la introducción de mejoras en el modelo pedagógico, la desconcentración, la gestión de la calidad en la investigación y la gestión de la información y la tecnología. Al ponerse en ejecución algunos asuntos concretos de la estrategia, se crea un ambiente de credibilidad en la estrategia y en las autoridades.

2 Se denomina Balanced Scorecard. El término se ha traducido como Anotador balanceado, Cuadro de mando integral y Cuadro de Comando.

3 Los indicadores se diseñan siguiendo un arreglo sistémico que permite observar simultáneamente cuatro dimensiones fundamentales: el aprendizaje institucional relacionado al desarrollo profesional de los funcionarios; el desarrollo de los procesos internos, tanto académicos como de gestión institucional, el aporte de la actividad académica a los estudiantes y a la sociedad y, finalmente, la perspectiva de lo financiero y lo presupuestario. 


\section{Conclusiones}

La estrategia basada en habilidades constituye un enfoque de utilidad para la gestión universitaria, ya que provee un patrón orientador tanto para la planificación como para los procesos de toma de decisiones. El hecho de basar la estrategia en un sistema de habilidades distintivas elaborado a partir de la visión, es congruente con la naturaleza de la organización universitaria, en la cual es importante asegurar el cumplimiento de su vocación y filosofía básica y donde se depende de las habilidades de los funcionarios. En la práctica, la elaboración de la estrategia es un proceso de aproximación gradual que se facilita mediante el análisis de adversidades y habilidades, lo cual realizado de manera participativa, coadyuva a crear un ambiente de credibilidad por parte de los actores involucrados.

El análisis político de la estrategia constituye una fase crítica en la cual el rigor del diseño estratégico puede pasar a un segundo plano frente al mayor énfasis puesto en la expresión de los intereses de los distintos sectores representados en los órganos de gobierno de la Universidad. Sin embargo, es una fase vital puesto que propicia el compromiso, la viabilidad, la sostenibilidad y el control político de la estrategia.

Los procesos de discusión de la estrategia por parte de las autoridades universitarias deben constituirse en una práctica habitual, ya que estimulan la comprensión del papel que cada quien desempeña en el logro de la visión y deja en claro la interdependencia de las distintas instancias académicas y administrativas para el logro de los objetivos estratégicos comunes. Asimismo, refuerza la conciencia de equipo y fortalece el principio de unidad por parte de las autoridades universitarias, aspectos relevantes para que una universidad pueda cumplir su misión en un momento histórico de gran dinamismo, complejidad e incertidumbre.

\section{Referencias}

Bricall. (2000). Informe Universidad 2000. Capítulo VIII. Gobierno y administración. Revista Vivat Academia. Alcalá. http://www2.uah.es/ vivatacademia/basedatos/bricall/cap8.htm

Boyett, J. y J. Boyett. 1999. Hablan los gurús. Bogotá. Norma.

Burdano, G. (1999). La educación superior en la segunda mitad del siglo XX. Los alcances del cambio en América Latina y el Caribe. Revista Iberoamericana de Educación (21). 
Dill, D. D. (1994). Defining the Common Good. The Use of Criteria in University Strategic Choice Making. $16^{\text {th }}$ Annual EAIR forum. Amsterdam.

Guy, N. (2001). Educación superior. Historia y política. Estudios comparativos sobre la universidad contemporánea. Barcelona. Gedisa.

Hammel, G. y Parlad. C. K. (1990). El propósito estratégico. HarvardDeusto Business Review (primer trimestre), 90 - 94.

Kaplan, R. y Norton, D. (2002). Cuadro de mando integral (The Balanced Scorecard). Barcelona. Gestión 2000.

Kaplan, R. y Norton, D. (2002). Cómo utilizar el cuadro de mando integral. Barcelona. Gestión 2000.

Massy, W. F. (1996). Reengineering Resource Allocation Systems. Resource Allocation in Higher Education, USA. Ann Arbor: University of Michigan Press, $15-47$.

Mintzberg, H. 1(988). Cómo modelar la estrategia. Harvard Deusto Business Review. (Segundo trimestre).

Mintzberg, H. (1988). La organización profesional. En Mintzber, H. y J. B. Quinn. (1988). El proceso estratégico. Conceptos, contextos y casos.

Miranda, R. y Carranza, F. (2002). Análisis de dificultades y habilidades distintivas. Universidad Nacional. Heredia, Costa Rica.

Porter. M. (1980). Estrategia competitiva. Técnicas de análisis de los sectores industriales y de la competencia. Traducido por Alfonso Vasseur Walls. $5^{\mathrm{a}}$ impresión. México, D.F. Continental.

Porter, M. (1997). ¿Qué es estrategia? Revista INCAE 10(1), 35 - 52.

Rama, C. (2002). La masificación universitaria y la emigración profesional de América Latina y el Caribe en la Sociedad del Conocimiento: algunas preguntas sin respuestas. Seminario "Migraciones e Integración Regional" Caracas SELA / CAF/UNESCO - ESALC. 
Richter, C. von. (2002). Palabras del Dr. Wolfgang von Kichter (GilL). Seminario: Desarrollo de la calidad en universidades de América Latina. México, Universidad Nacional Autónoma de México, 8 - 9.

Ruiz, A. (2001). La educación superior en Costa Rica. Tendencias y retos en un nuevo escenario histórico. San José. Editorial de la Universidad de Costa Rica.

Stalk, G., P. Evans y L. Shulman. (1992). Competir en habilidades, clave de la nueva estrategia empresarial. Harvard Deusto Business Review. Pp. 44-56.

Vucht Tijssen, van L.( 2002). Un programa estratégico para la investigación en la Universidad Nacional de Costa Rica. Universidad de Utrecht. Holanda.

UNESCO. (1998). Declaración mundial sobre la educación superior en el siglo XXI: visión y acción. Conferencia Mundial Sobre La Educación Superior. París. http://www. unesco.org/education/educprog/wche/ declaration_spa.htm

UNESCO. (1998). Marco de acción prioritaria para el cambio y el desarrollo de la educación superior. Conferencia Mundial Sobre La Educación Superior. París. http://www.unesco.org/education/educprog/wche/ declaration_spa.htm

Universidad Nacional. (2004). Informe sobre planes de estudio ofrecidos por grado académico, según Unidad Académica, a diciembre 2003.

Universidad Nacional (2004). Plan Global Institucional 2004 -2014. 